\title{
A Penetrating Lung Injury by a Feeding Tube
}

\author{
Seok Won Lee', Dong Hun Kim¹, Ye Rim Chang', Jeongseok Yun', Seokho Choi', \\ Sung Wook Chang', Jung-Ho Yun ${ }^{3}$ \\ ${ }^{1}$ Department of Trauma Surgery, ${ }^{2}$ Department of Thoracic and Cardiovascular Surgery, ${ }^{3}$ Department of Neurosurgery, Trauma Center, \\ Dankook University Hospital, Cheonan, Republic of Korea
}

Negligent perforation in the tracheobronchial tree during feeding tube insertion is not uncommon in the elderly, neurologically impaired, and seriously ill patients. Risk factors for feeding tube misplacement include altered mental status, sedation, critical illness, intubation, absent cough reflex, difficult or repeat tube placement, noncompliant behavior, and anatomic abnormalities. Here, we report a case of iatrogenic lung injury due to a displaced 12-Fr feeding tube in a 68-year-old male with traumatic brain and cervical cord injuries.

(Trauma Image Proced 2018(2):50-52)

Key Words: Gastric Feeding Tube; Displacement; Central Nervous System; Trauma

\section{CASE}

A 68-year-old male sustained multiple facial bone fractures, traumatic subarachnoid hemorrhage, and spinal cord injury at the 5th cervical spine level following a traffic accident, with an injury severity score of 36 . He underwent tracheostomy for continuous ventilator care on hospital day 10 , because of diaphragmatic paralysis and drowsy mental state. A 16-Fr. Levin tube was inserted to provide enteral nutrition, but was sometimes clogged; therefore, on hospital day 22, it was replaced with a 12-Fr. Kangaroo ${ }^{\mathrm{TM}}$ feeding tube, which is originally inserted with an inner guide-wire that is removed after completing the insertion. A supine chest $\mathrm{X}$-ray performed after inserting the feeding tube revealed malposition in the right thorax with hemothorax (Fig. 1). A chest computed tomography scan revealed that the feeding tube had penetrated the right lower bronchial branch and is located in the right pleural space with hemopneumothorax (Fig. 2). The feeding tube was removed after performing right thoracostomy followed by bronchoscopy to determine the need for surgical treatment. Bronchoscopy revealed no endobronchial lesion within the visible whole range, except for the small amount of fresh blood in the bronchial branch at the right lower lobe (Fig. 3). Air leakage and drain volume via a chest tube gradually decreased, and the tube was removed 10 days after performing thoracostomy (Fig. 4). The patient recovered without any lung sequela.

\section{DISCUSSION}

Feeding tube misplacement is a potentially fatal and

Received: September 19, 2018 Revised: October 19, 2018 Accepted: October 19, 2018

Correspondence to: Dong Hun Kim, Department of Trauma Surgery, Trauma Center, Dankook University Hospital, 201 Manghyang-ro, Dongnam-gu, Cheonan 31116, Republic of Korea

Tel: 82-41-550-7661, Fax: 82-41-550-0039, E-mail: saint7331@gmail.com

Copyright (c) 2018 Korean Association for Research, Procedures and Education on Trauma. All rights reserved.

@This is an open-access article distributed under the terms of the Creative Commons Attribution Non-Commercial License (http://creativecommons.org/ licenses/by-nc/4.0) which permits unrestricted noncommercial use, distribution, and reproduction in any medium, provided the original work is properly cited 


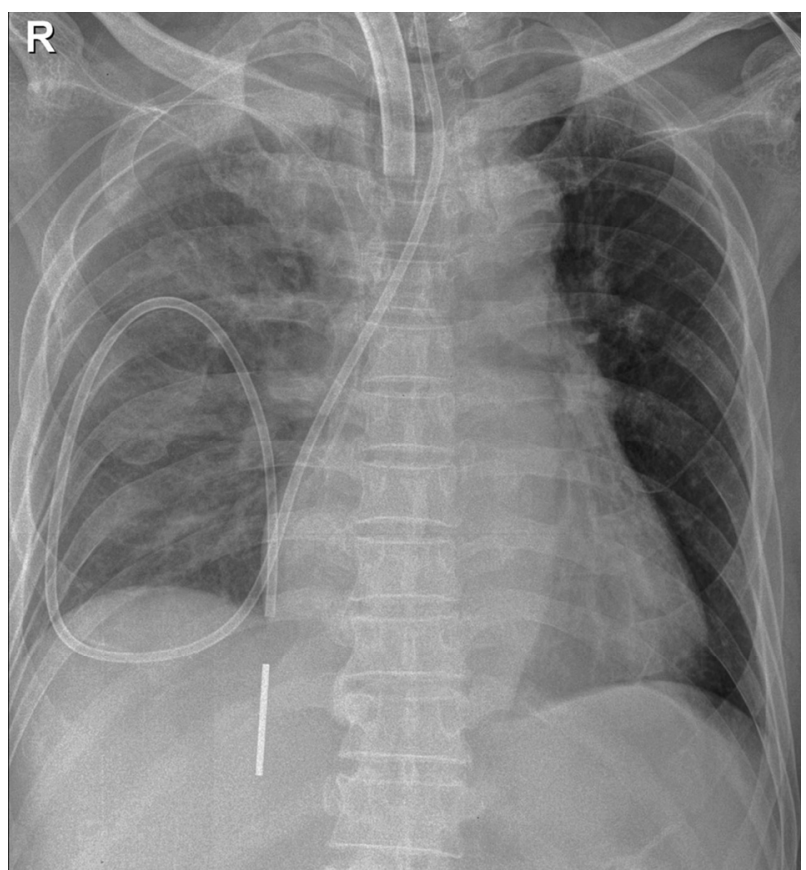

Fig. 1. A chest radiograph showing feeding tube malposition through the tracheobronchial tree in the right hemithorax with hemothorax.

underestimated problem. Misplacements usually occur in specific patient groups: $60 \%$ during mechanical ventilation, $74 \%$ in critical illness, and $96 \%$ in depressed consciousness $(1,2)$. Approximately $1.2 \%-2 \%$ of all blind feeding tube insertions result in airway misplacement, $0.3 \%-0.7 \%$ cause pulmonary injury (pneumothorax, hemopneumothorax, hydrothorax, empyema, and pneumonia), and $0.1 \%-0.3 \%$ lead to death (3). In this case, a tracheostomy tube with an inflated cuff was properly placed and did not prevent the feeding tube from entering the trachea. However, in patients on mechanical ventilation and with positive airway pressures, a prophylactic chest tube should be placed before removing the misplaced tube, because of the precluding development of a life-threatening tension pneumothorax (4).

The following are some modalities that are used to confirm feeding tube placement: air insufflation during abdominal auscultation, fluid aspiration and $\mathrm{pH}, \mathrm{CO}_{2}$ detection via capnography or colorimetry, electromagnetic trace, endoscopy and fluoroscopy, or X-ray (5). Postplacement radiograph is the gold standard method

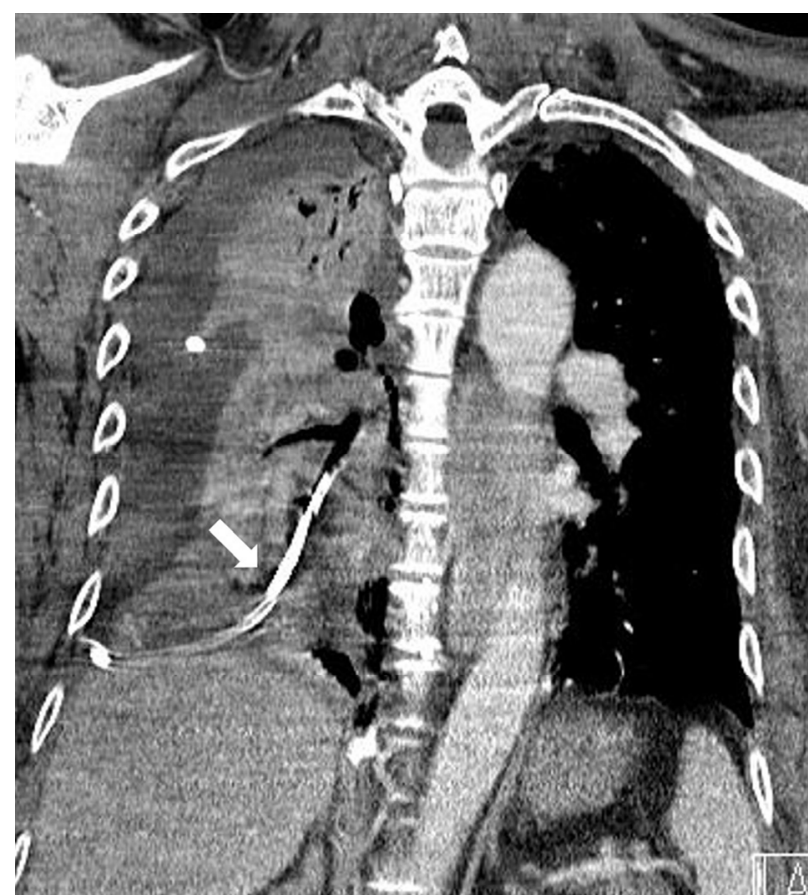

Fig. 2. A chest computed tomography showing feeding tube misplacement where the tube penetrated the right lower bronchial branch and lung parenchyma (indicated by white arrow) and located in the right pleural space with hemopneumothorax.

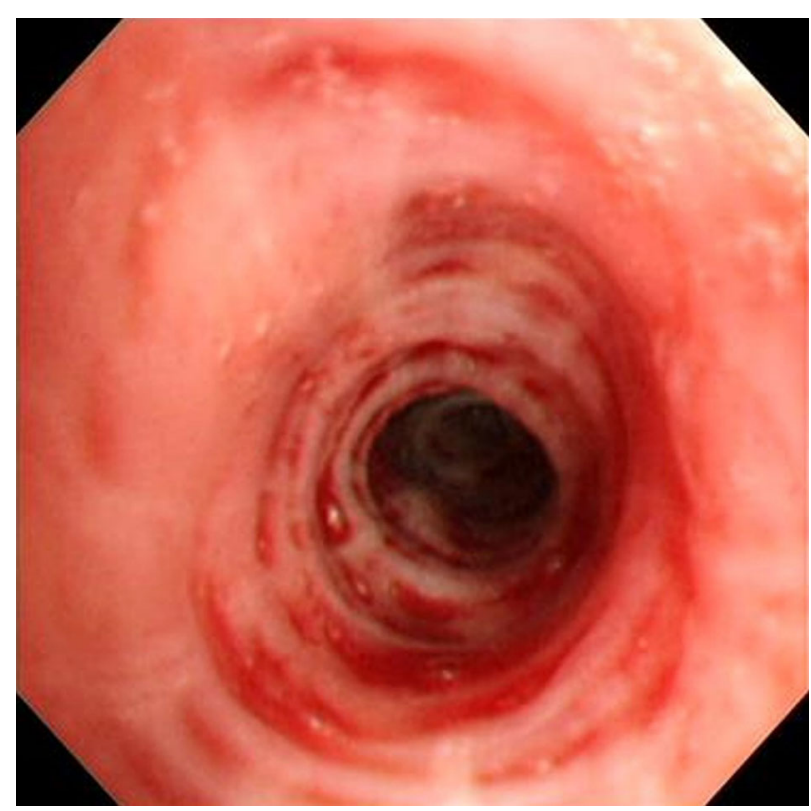

Fig. 3. Bronchoscopy showing no visible endobronchial lesion in the bronchial branch (B8) of the right lower lobe stained with small amount of fresh blood. 

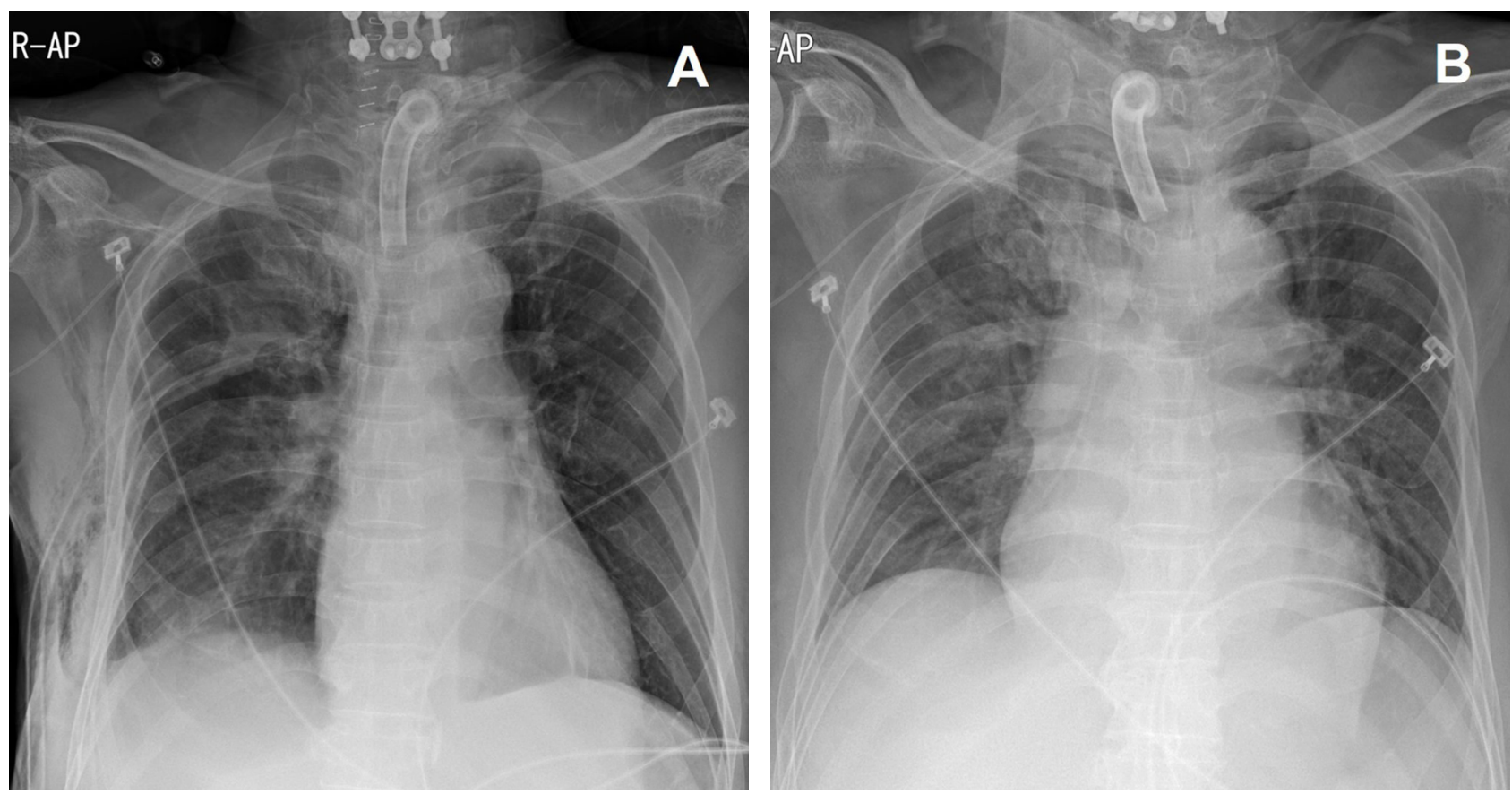

Fig. 4. Chest radiographs showing $(A)$ right thoracostomy state after removing the misplaced feeding tube and (B) normal expansion of the right lung without any sequela associated with feeding tube misplacement after the chest tube removal 10 days later.

used to confirm tube position. Thorough and early recognition of misplacement and timely management of consequent complications should be implemented to decrease morbidity and even mortality in enteral feeding tube placement.

\section{Conflicts of Interest Statement}

None of authors have a conflict of interest.

\section{REFERENCE}

1. Sorokin R, Gottlieb JE. Enhancing patient safety during feeding tube insertion. A review of more than 2000 insertions. J Parenter Enteral Nutr. 2006;30:440-5.
2. Sparks DA, Chase DM, Coughlin LM, Perry E. Pulmonary complications of 9931 narrow-bore nasoenteric tubes during blind placement: a critical review. J Parenter Enteral Nut. 2011;35:625-3.

3. Krenitsky J. Nutrition issues in gastroenterology, series no. 93: blind bedside placement of feeding tubes: treatment or threat? Pract Gastroenterol. 2011;35(3):32-42.

4. Odocha O, Lowery RC Jr, Mezghebe HM, Siram SM, Warner OG. Tracheopleuropulmonary injuries following enteral tube insertion. J Natl Med Assoc. 1989 Mar;81(3): 275-81.

5. Taylor SJ. Confirming nasogastric feeding tube position versus the need to feed. Intensive Crit Care Nurs. 2013 Apr;29(2):59-69. 